ADP-95-36/T190

\title{
Effect of Nucleon Structure Variation in Super-allowed Fermi Beta-decay
}

\author{
K. Saito \\ Physics Division, Tohoku College of Pharmacy \\ Sendai 981, Japan \\ and \\ A. W. Thomas E \\ Department of Physics and Mathematical Physics \\ and \\ Institute for Theoretical Physics \\ University of Adelaide, 5005, Australia
}

\begin{abstract}
There is a well known anomaly between the value of the Fermi decay constant extracted from super-allowed Fermi beta-decay of nuclear isotriplets and that required by unitarity of the Cabibbo-Kobayashi-Maskawa matrix. This discrepancy remains at the level of a few tenths of a percent after the most rigorous investigation of conventional nuclear and radiative corrections. Within the framework of the quark-meson coupling model of nuclear matter, which has been previously applied successfully to phenomena such as nuclear saturation and nuclear charge symmetry violation, we show that it is possible to understand a significant fraction of the observed anomaly.
\end{abstract}

\footnotetext{
${ }^{1}$ ksaito@nucl.phys.tohoku.ac.jp

${ }^{2}$ athomas@physics.adelaide.edu.au
} 
It is clearly very important to refine our understanding of the weak coupling to quarks as much as possible. Testing the unitarity of the Cabibbo-Kobayashi-Maskawa (CKM) matrix is one of the more challenging aspects of this general problem. In particular, the most accurate experimental measurement of the vector coupling constant in nuclear beta-decay comes from super-allowed $0^{+}-0^{+}$transitions between nuclear isotriplet states. However, in order to relate these precise measurements to the quark-level vector coupling, $V_{u d}$, one needs to apply a number of small nuclear structure corrections [1] in addition to the relatively standard radiative corrections [2]. Despite intensive study of these nuclear "mismatch" corrections [3, 4, 5, 6] there remains a systematic difference of a few tenths of a percent between the value of $V_{u d}$ inferred from the vector coupling measured in muon decay, $G_{\mu}$, and unitarity of the CKM matrix and those determined from the nuclear $f t$ values. For recent summaries we refer to the reviews of Wilkinson [7] and Towner and Hardy [8], and also to the recent report by Savard et al. [9] of accurate data on ${ }^{10} \mathrm{C}$.

Until now the nuclear corrections have been explored within the framework of conventional nuclear theory with point-like nucleons. Of course, for the nucleon itself there has been considerable investigation of the effect on the vector form-factor of the breaking of CVC caused by the small $u-d$ mass difference in QCD. The Ademollo-Gatto theorem [10, 11] tells us that any corrections must be of second order in $\left(m_{d}-m_{u}\right)$ - a result that has survived [12, 13] suggestions that it might fail because of $\rho-\omega$ mixing [14]. While this is necessarily very small, the measurements of $V_{u d}$ and $G_{\mu}$ are also extremely precise. Thus we have been led to ask whether this small nuclear discrepancy might be associated with a change in the degree of non-conservation of the vector current caused by nuclear binding.

In order to investigate whether nuclear binding might influence the Fermi decay constant of the nucleon itself one needs a model of nuclear structure involving explicit quark degrees of freedom which nevertheless provides an acceptable description of nuclear binding and saturation. The quark-meson coupling (QMC) model of Guichon [15] seems ideally suited to the problem. In this model, nuclear matter consists of non-overlapping 
nucleon bags bound by the self-consistent exchange of $\sigma$ and $\omega$ mesons in the mean-field approximation. It has been extended by Yazaki et al. [16] to include a centre of mass correction and by the present authors to include the $\rho$ and an isovector-scalar meson (the

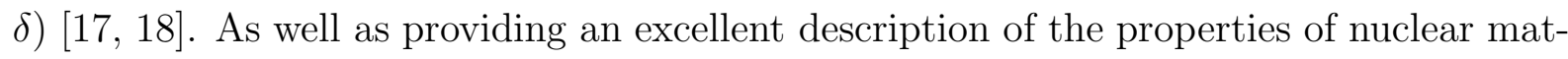
ter, it has been applied successfully to the calculation of nuclear structure functions [19] and (allowing for a difference in the quark masses, $m_{u} \neq m_{d}$ ) to the problem of the Okamoto-Nolen-Schiffer anomaly [20] in mirror nuclei [18]. Furthermore, the relationship between the QMC model and Quantum Hadrodynamics [21] has been investigated. The fascinating result is that for infinite nuclear matter the two approaches can be written in an identical form, except for the appearance of the quark-scalar density in the selfconsistency condition for the scalar field [17, 22]. The simplicity of this finding suggests that it may be rather more general than the specific model within which it was derived.

We shall briefly review the main features of the model with unequal quark masses before turning to the specific problem of the Fermi form-factor. Suppose that the meanfield values for the $\sigma, \omega$ (the time component), $\rho$ (the time component in the third direction of isospin) and $\delta$ (in the third direction of isospin) fields, in uniformly distributed nuclear matter with $N \neq Z$, are $\bar{\sigma}, \bar{\omega}, \bar{b}$ and $\bar{\delta}$, respectively. The nucleon is described by the static spherical MIT bag in which quarks interact (self-consistently) with those mean fields. Then the Dirac equation for a quark field, $\psi$, in a bag is given by

$$
\left[i \gamma \cdot \partial-\left(m_{i}-V_{\sigma}-\frac{1}{2} \tau_{z} V_{\delta}\right)-\gamma^{0}\left(V_{\omega}+\frac{1}{2} \tau_{z} V_{\rho}\right)\right] \psi_{i / j}=0
$$

where $V_{\sigma}=g_{\sigma}^{q} \bar{\sigma}, V_{\omega}=g_{\omega}^{q} \bar{\omega}, V_{\rho}=g_{\rho}^{q} \bar{b}$ and $V_{\delta}=g_{\delta}^{q} \bar{\delta}$ with the quark-meson coupling constants, $g_{\sigma}^{q}, g_{\omega}^{q}, g_{\rho}^{q}$ and $g_{\delta}^{q}$. The subscripts, $i$ and $j$, denote the $i$-th quark $(i=u$ or $d)$ in the proton or neutron $(j=p$ or $n)$.

The normalized, ground state wave function for a quark in the nucleon is then given by

$$
\psi_{i / j}(\vec{r}, t)=\mathcal{N}_{i / j} \exp \left[-i \epsilon_{i / j} t / R_{j}\right]\left(\begin{array}{c}
j_{0}\left(x_{i} r / R_{j}\right) \\
i \beta_{i / j} \vec{\sigma} \cdot \hat{r} j_{1}\left(x_{i} r / R_{j}\right)
\end{array}\right) \frac{\chi_{i}}{\sqrt{4 \pi}}
$$


where

$$
\begin{gathered}
\epsilon_{i / j}=\Omega_{i / j}+R_{j}\left(V_{\omega} \pm \frac{1}{2} V_{\rho}\right), \text { for a }\left(\begin{array}{l}
u \\
d
\end{array}\right) \text { quark } \\
\mathcal{N}_{i / j}^{-2}=2 R_{j}^{3} j_{0}^{2}\left(x_{i}\right)\left[\Omega_{i / j}\left(\Omega_{i / j}-1\right)+R_{j} m_{i}^{\star} / 2\right] / x_{i}^{2} \\
\beta_{i / j}=\sqrt{\left(\Omega_{i / j}-R_{j} m_{i}^{\star}\right) /\left(\Omega_{i / j}+R_{j} m_{i}^{\star}\right)}
\end{gathered}
$$

with $\Omega_{i / j}=\sqrt{x_{i}^{2}+\left(R_{j} m_{i}^{\star}\right)^{2}}$ and $\chi_{i}$ the quark spinor. The effective quark mass, $m_{i}^{\star}$, is defined by

$$
m_{i}^{\star}=m_{i}-\left(V_{\sigma} \pm \frac{1}{2} V_{\delta}\right), \text { for a }\left(\begin{array}{l}
u \\
d
\end{array}\right) \text { quark. }
$$

The linear boundary condition at the bag surface determines the eigenvalue $x_{i}$.

Taking the spin-flavor wave function for the nucleon to have the usual SU(6) form, the nucleon energy is given by $E_{b a g}^{j}+3 V_{\omega} \pm \frac{1}{2} V_{\rho}$ for $\left(\begin{array}{l}p \\ n\end{array}\right)$, where the bag energy is

$$
E_{b a g}^{j}=\frac{\sum_{i} n_{i / j} \Omega_{i / j}-z_{0}}{R_{j}}+\frac{4}{3} \pi B R_{j}^{3},
$$

$B$ is the bag constant and $z_{0}$ is a phenomenological parameter initially introduced to account for zero-point motion. Here we use it to correct for spurious c.m. motion as well - rather than following the more elaborate procedure of Ref. [16. The reason is that, as illustrated by the Ademollo-Gatto theorem, the deviation of the vector form-factor from unity is a very subtle effect. This method is the only one in which we have been able to guarantee that in the free nucleon case the deviation is proportional to $\left(m_{d}-m_{u}\right)^{2}$. The effective nucleon mass, $M_{j}^{\star}$, in nuclear matter is given by minimizing eq.(7) with respect to $R_{j}$.

To see the sensitivity of our results to the bag radius of the free nucleon, we choose $m_{u}=5 \mathrm{MeV}$ and vary the parameters, $B, z_{0}$ and $m_{d}$, to fit the physical proton and neutron masses for several values of the average, free bag radius $R_{0}(=0.6,0.8,1.0 \mathrm{fm})$. Since the electromagnetic (EM) self-energies for $p$ and $n$ contribute to the masses we adjust the parameters to fit the bare proton mass, $M_{p}=938.272-0.63 \mathrm{MeV}$, and the bare neutron mass, $M_{n}=939.566+0.13 \mathrm{MeV}$, where $+0.63 \mathrm{MeV}$ and $-0.13 \mathrm{MeV}$ are the EM self-energies for $p$ and $n$, respectively [23]. We then find that $B^{1 / 4}=(210.7,169.8$, 
143.6) $\mathrm{MeV}, z_{0}=(4.011,3.305,2.600)$, and $m_{d}=(9.252,9.242,9.233) \mathrm{MeV}$ for $R_{0}=$ $(0.6,0.8,1.0) \mathrm{fm}$, respectively. It is crucial for the present application that the bag radius of the proton is a little smaller than that of the neutron in matter and that this difference increases slightly with density.

At the present time the extension of the QMC model to finite nuclei is not complete [24]. In particular, the variation of the scalar and vector fields across the finite size of the nucleon presents quite a challenge. Thus, for the present we are constrained to discuss the problem within the framework of infinite nuclear matter. In this case we take the Fermi momenta for protons and neutrons to be $k_{F_{p}}$ and $k_{F_{n}}$, respectively. These are defined by $\rho_{p}=k_{F_{p}}^{3} /\left(3 \pi^{2}\right)$ and $\rho_{n}=k_{F_{n}}^{3} /\left(3 \pi^{2}\right)$, where $\rho_{p}$ and $\rho_{n}$ are the densities of $p$ and $n$, respectively, and the total baryon density, $\rho_{B}$, is given by $\rho_{p}+\rho_{n}$. The $\omega$ field is determined by baryon number conservation, and the $\rho$ mean-field by the difference in proton and neutron densities ( $\rho_{3}$ below). On the other hand, the scalar mean-fields, $\bar{\sigma}$ and $\bar{\delta}$, are given by self-consistency conditions (SCCs) [17, 18]. Since the $\rho$ field value is given by $\bar{b}=g_{\rho} \rho_{3} /\left(2 m_{\rho}^{2}\right)$, where $g_{\rho}=g_{\rho}^{q}$ and $\rho_{3}=\rho_{p}-\rho_{n}$, the total energy per nucleon, $E_{t o t}$, can be written

$$
E_{\text {tot }}=\frac{2}{\rho_{B}(2 \pi)^{3}} \sum_{j=p, n} \int^{k_{F_{j}}} d \vec{k} \sqrt{M_{j}^{\star 2}+\vec{k}^{2}}+\frac{m_{\sigma}^{2}}{2 \rho_{B}} \bar{\sigma}^{2}+\frac{m_{\delta}^{2}}{2 \rho_{B}} \bar{\delta}^{2}+\frac{g_{\omega}^{2}}{2 m_{\omega}^{2}} \rho_{B}+\frac{g_{\rho}^{2}}{8 m_{\rho}^{2} \rho_{B}} \rho_{3}^{2},
$$

where $g_{\omega}=3 g_{\omega}^{q}$.

We determine the coupling constants, $g_{\sigma}^{2} / 4 \pi=(22.62,20.78,19.47)$, where $g_{\sigma}=3 g_{\sigma}^{q}$, and $g_{\omega}^{2} / 4 \pi=(5.547,4.637,3.979)$ for $R_{0}=(0.6,0.8,1.0) \mathrm{fm}$, respectively, so as to fit the binding energy $(-16 \mathrm{MeV})$ and the saturation density $\left(\rho_{0}=0.17 \mathrm{fm}^{-3}\right)$ for equilibrium nuclear matter. It is important to stress that the $\delta$ plays an insignificant role in our present calculation, while the $\rho$ has no influence in symmetric matter. Nevertheless, for the record we note that $g_{\delta}^{2} / 4 \pi$ is chosen to be 2.82 [25] (where $g_{\delta}=g_{\delta}^{q}$ ) and the $\rho$ meson coupling constant, $g_{\rho}^{2} / 4 \pi$, is chosen to be $(5.223,5.340,5.423)$ for $R_{0}=(0.6,0.8,1.0) \mathrm{fm}$, respectively, so as to reproduce the bulk symmetry energy of nuclear matter, $33.2 \mathrm{MeV}$. One of the important consequences of including the internal structure of the nucleon is that the model gives a very respectable value for the nuclear incompressibility, around 


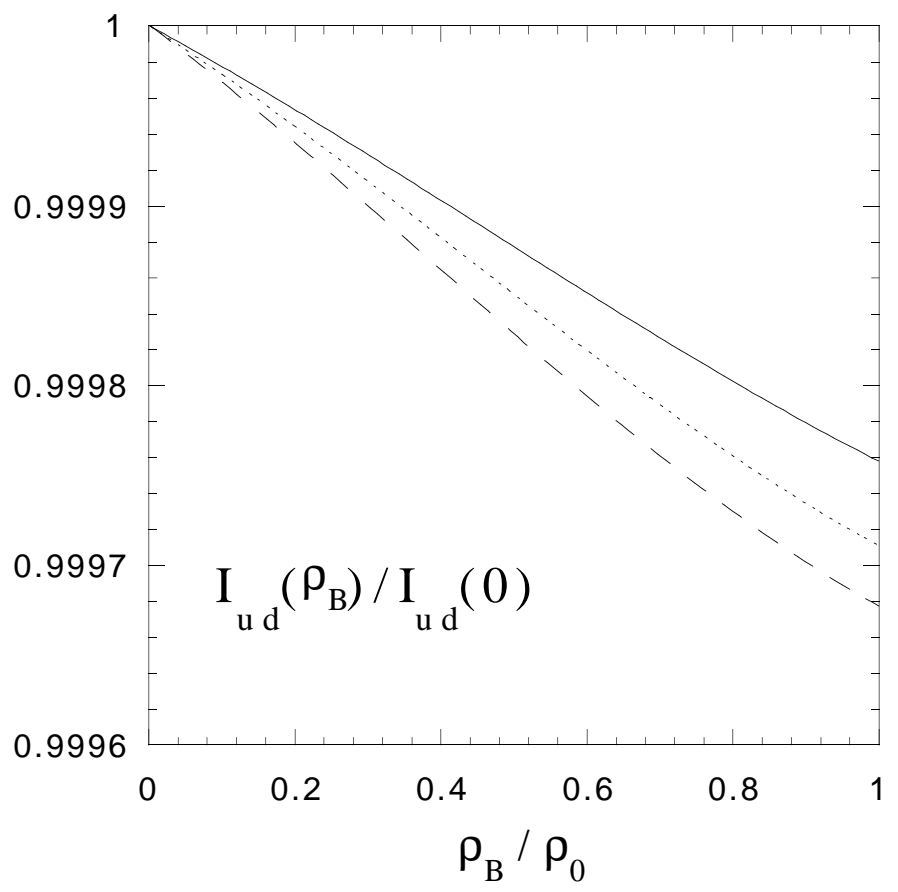

Figure 1: $I_{u d}\left(\rho_{B}\right) / I_{u d}(0)$ as a function of the nuclear density. The solid, dotted and dashed curves are for $R_{0}=0.6,0.8$ and $1.0 \mathrm{fm}$, respectively.

$200 \sim 300 \mathrm{MeV}$.

Armed with an acceptable, quark-based model of nuclear matter we can now investigate the variation with density of the quark vector current matrix element. For Fermi beta-decay we need the matrix elements

$$
I_{i i^{\prime}}\left(\rho_{B}\right)=\int_{B a g} d V \psi_{i / p}^{\dagger} \psi_{i^{\prime} / n}
$$

with $i^{\prime}=d$ and $i=u$ for the $d \rightarrow u$ conversion and $i=i^{\prime}=u$ or $d$ for the two spectator quarks. As the radius of the proton and neutron are different we integrate over the common volume.

As remarked earlier, the first test of the consistency of the calculation is that at zero density the deviation of the vector form-factor from unity is quadratic in $m_{d}-m_{u}$. By a numerical check we can see that this is indeed the case .

In fig.11 we show the ratio $I_{u d}\left(\rho_{B}\right) / I_{u d}(0)$, for our three choices of the free (average) bag 
radius (in symmetric nuclear matter), as a function of $\rho_{B}$. We also give full details of those values at several nuclear densities in table 1. The decrease in $I_{i i^{\prime}}$ as the density increases is a direct consequence of the increasing difference between the proton and neutron radii - that is the smaller volume of overlap. From the figure and the table, as the density

Table 1: Variations with density of $I_{i i^{\prime}}$ for three bag radii.

\begin{tabular}{ccccc}
\hline$R_{0}(\mathrm{fm})$ & $\rho_{B} / \rho_{0}$ & $I_{u d}$ & $I_{u u}$ & $I_{d d}$ \\
\hline \multirow{2}{*}{0.6} & 0 & 0.999993 & 0.999995 & 0.999995 \\
& 0.5 & 0.999869 & 0.999870 & 0.999871 \\
& 1.0 & 0.999751 & 0.999751 & 0.999752 \\
\hline \multirow{2}{*}{0.8} & 0 & 0.999991 & 0.999994 & 0.999994 \\
& 0.5 & 0.999840 & 0.999842 & 0.999843 \\
& 1.0 & 0.999702 & 0.999703 & 0.999705 \\
\hline \multirow{2}{*}{1.0} & 0 & 0.999987 & 0.999992 & 0.999992 \\
& 0.5 & 0.999814 & 0.999817 & 0.999819 \\
& 1.0 & 0.999664 & 0.999666 & 0.999669 \\
\hline
\end{tabular}

increases from zero, we see that the deviation of $I_{i i^{\prime}}\left(\rho_{B}\right) / I_{i i^{\prime}}(0)$ from unity is roughly linear in $\rho_{B}$. (The deviation is also roughly linear in $m_{d}-m_{u}$.) We can then summarise the results as:

$$
\frac{I_{i i^{\prime}}\left(\rho_{B}\right)}{I_{i i^{\prime}}(0)} \simeq 1-a_{i i^{\prime}} \times\left(\frac{\rho_{B}}{\rho_{0}}\right),
$$

with $a_{i i^{\prime}} \simeq(2.4,2.9,3.3) \times 10^{-4}$ for $R_{0}=(0.6,0.8,1.0) \mathrm{fm}$, respectively (for any combination of $\left.i i^{\prime}\right)$.

The evaluation of $f t$-values involves the inverse of the product of $I_{u d}, I_{u u}$ and $I_{d d}$ squared. Since for a given, free (average) radius of the bag each of these matrix elements decreases by roughly the same amount, the fractional increase in the $f t$-value with density 
is therefore

$$
\frac{f t\left(\rho_{B}\right)}{f t(0)} \simeq 1+b \times\left(\frac{\rho_{B}}{\rho_{0}}\right),
$$

with $b$ approximately six times the decrease in each integral - i.e. $b \simeq(1.5,1.8,2.0) \times 10^{-3}$ for $R_{0}=(0.6,0.8,1.0) \mathrm{fm}$. Thus the increase in the $f t$-value at $\rho_{0} / 2$ ranges from $0.075 \%$ to $0.10 \%$, while at $\rho_{0}$ it lies between $0.15 \%$ and $0.20 \%$. This is to be compared with a violation of unitarity of the CKM matrix of $0.35 \pm 0.15 \%$ in the most recent analysis of Towner and Hardy [8].

It is not possible to draw unambiguous conclusions from a comparison of theoretical results in infinite nuclear matter with data from finite nuclei. At $\rho_{0} / 2$ the calculation suggests a reduction in the violation of unitarity by about $1 / 3$, while at $\rho_{0}$ a correction as big as $0.2 \%$ brings the discrepancy back to only one standard deviation. We also note that this result is rather sensitive to the proton fraction, $f_{p}=\rho_{p} / \rho_{B}$. The overlap integrals with $f_{p}$ less (greater) than 0.5 decrease more slowly (rapidly) than in the symmetric case.

Before concluding our discussion it might be helpful to make a qualitative observation concerning the validity of our results. The essential physics involved in our calculation is charge symmetry violation, in particular, the fact that in nuclear matter the confining potential felt by a quark in a proton is not the same as that felt by a quark in a neutron. We have already explained that a relativistic field theory only yields the right order of magnitude for nuclear charge symmetry breaking if the relevant mass scale involves quarks rather than nucleons [26]. In this sense the ONS anomaly may prove to be something of a "smoking gun" for quark degrees of freedom in nuclei. This is even more obvious here; it is only because the nuclear charge symmetry violation occurs at the quark level that it can produce a deviation of the vector form factor of the bound nucleon from its free value.

In summary, within a specific, relativistic quark-meson coupling model of nuclear matter we have found that the change of the internal structure of the nucleon itself produces a correction to the Fermi beta-decay form-factor of the sign and order of magnitude required for consistency with the unitarity of the Cabibbo-Kobayashi-Maskawa matrix. It 
is important that the model dependence of this result be further examined as soon as possible - although we have given arguments why the qualitative result may be quite general. Finally we note that the present model deals only with nuclear matter and it is vital that the formalism be extended so that we can apply the model quantitatively in finite nuclei.

We would like to thank Peter Jackson for raising this issue with one of us during the celebration of Erich Vogt's 65th birthday at TRIUMF last December. We would also like to acknowledge helpful discussions with T. Hatsuda, I. Towner and D. Wilkinson during WEIN'95. This work was supported by the Australian Research Council. 


\section{References}

[1] D.H. Wilkinson, Z. Phys. A348 (1994) 129.

[2] I.S. Towner, Phys. Lett. B333 (1994) 13;

W.J. Marciano and A. Sirlin, Phys. Rev. Lett. 61 (1988) 1815.

[3] D.H. Wilkinson, Phys. Lett. B65 (1976) 9;

I.S. Towner, J.C. Hardy and M. Harvey, Nucl. Phys. A284 (1977) 269.

[4] W.E. Ormond and B.A. Brown, Nucl. Phys. A440 (1985) 274; Phys. Rev. Lett. 62 (1989) 866; ORNL preprint, ORNL-CTP-95-05, nucl-th/9504017.

[5] F.C. Barker, Nucl. Phys. A537 (1992) 134.

[6] D.H. Wilkinson, Nucl. Phys. A511 (1990) 301.

[7] D.H. Wilkinson, TRIUMF preprints TRI-PP-94-95 (Oct. 1994) and TRI-PP-94-102 (Nov. 1994).

[8] I.S. Towner and J.C. Hardy, Chalk River preprint, nucl-th/9504015 (April 1995).

[9] G. Savard et al., Phys. Rev. Lett. 74 (1995) 1521.

[10] M. Ademollo and R. Gatto, Phys. Rev. Lett. 13 (1964) 264.

[11] R. Behrends and A. Sirlin, Phys. Rev. Lett. 4 (1960) 186.

[12] J.F. Donoghue and D. Wyler, Phys. Lett. B241 (1990) 243.

[13] N. Paver and Riazuddin, Phys. Lett. B260 (1991) 421.

[14] G. Lopez Castro and J. Pestieau, Phys. lett. B203 (1988) 315.

[15] P.A.M. Guichon, Phys. Lett. B200 (1988) 235.

[16] S. Fleck, W. Bentz, K. Shimizu and K. Yazaki, Nucl. Phys. A510 (1990) 731. 
[17] K. Saito and A.W. Thomas, Phys. Lett. B327 (1994) 9.

[18] K. Saito and A.W. Thomas, Phys. Lett. B335 (1994) 17.

[19] K. Saito, A. Michels and A.W. Thomas, Phys. Rev. C46 (1992) R2149;

A.W. Thomas, K. Saito and A. Michels, Aust. J. Phys. 46 (1993) 3;

K. Saito and A.W. Thomas, Nucl. Phys. A574 (1994) 659.

[20] K. Okamoto, Phys. Lett. 11 (1964) 150;

J.A. Nolen, Jr. and J.P. Schiffer, Ann. Rev. Nucl. Sci. 19 (1969) 471.

[21] B.D. Serot and J.D. Walecka, Advan. Nucl. Phys. vol.16 (1986) 1.

[22] K. Saito and A.W. Thomas, Univ. of Adelaide, preprint ADP-95-32/T186, nucl th/9506003.

[23] J. Gasser and H. Leutwyler, Phys. Rep. C87 (1982) 77.

[24] P.A.M. Guichon, K. Saito and A.W. Thomas, in preparation.

[25] M. Machleidt, K. Holinde and Ch. Elster, Phys. Rep. C149 (1987) 1;

J. Haidenbauer, K. Holinde and A.W. Thomas, Phys. Rev. C45 (1992) 952.

[26] A.W. Thomas and K. Saito, "The Role of Nucleon Structure in Nuclear Physics", in Proc. Int. Conf. on Physics with GeV Beams, eds. H. Machner and K. Sistemich (World Scientific, Singapore, 1995). 